\title{
Construction of Low-density Parity-check Codes from Kirkman Triple Systems
}

\author{
Sarah J. Johnson and Steven R. Weller \\ Department of Electrical and Computer Engineering \\ University of Newcastle, Callaghan, NSW 2308, Australia
}

\begin{abstract}
Gallager introduced low-density parity-check (LDPC) codes in 1962, presenting a construction method to randomly allocate bits in the parity-check matrix subject to certain structural constraints. Since then improvements have been made to Gallager's construction method and some analytic constructions for LDPC codes have recently been presented. However analytically constructed LDPC codes comprise only a very small subset of possible codes and as a result LDPC codes are still, for the most part, constructed randomly. This paper extends the class of LDPC codes that can be systematically generated by presenting a construction method for regular LDPC codes based on combinatorial designs known as Kirkman triple systems. That is, we construct $(3, \rho)$-regular codes whose Tanner graph is free of 4-cycles for any integer $\rho$.
\end{abstract}

\section{INTRODUCTION}

Low-density parity-check (LDPC) codes were discovered by Gallager [1] in 1962 and have recently been rediscovered [2], [3]. LDPC codes are designed by specifying a sparse paritycheck matrix $H$ so that the relationship between code bits and parity-check sums can be adjusted to optimize the flow of information in the decoding process. Gallager presented iterative decoding algorithms whose complexity remains linear in the block length [4]. Recently, it has been shown that the encoding complexity of LDPC codes can also remain linear in the block length [5]. When iteratively decoded using belief propagation, also known as sum-product decoding, [6], [7], LDPC codes can perform remarkably close to the Shannon limit [2].

A Tanner graph displays the relationship between codeword bits and parity checks and is a useful way to describe LDPC codes [3]. Each of the $n$ code bits, and $m$ parity checks in $H$ are represented by a vertex in the graph. A graph edge joins a code bit vertex to the vertices of the parity checks that include it. The graph is bipartite since no parity check is connected to another by an edge, and no two code bits are connected. The number of edges connected to a code bit vertex is the degree of that code bit, which is simply the number of parity check equations that include it. Likewise, the degree of a parity check vertex is the number of bits in the parity check equation. An LDPC code is said to be $(\gamma, \rho)$-regular if the degrees of all code bit vertices are $\rho$ and the degrees of all parity check vertices are $\gamma$

It is known that the iterative sum-product decoding algorithm converges to the optimal solution provided the Tanner graph of the code satisfies a structural constraint-namely, that it be free of cycles [8], [9]. A cycle in a Tanner graph is a

Work supported in part by a CSIRO Telecommunications \& Industrial Physics postgraduate scholarship, and by the Centre for Integrated Dynamics and Control. sequence of connected code bits and check sums which start and end at the same vertex in the graph and contain no other vertices more than once. The length of the cycle is simply the number edges it contains and the girth of a Tanner graph is the size of its smallest cycle.

The existence of short cycles in the Tanner graph prevents an exact error-probability analysis of iterative decoding procedures, and the shorter are the cycles in the graph, the sooner the analysis breaks down. Various improvements have been made to Gallager's original construction method to avoid cycles and obtain the desired column and row weights [2], [6], [10]. However, randomly constructed LDPC codes have to date largely relied on the sparsity of the parity-check matrix to avoid short cycles in the Tanner graph. When the block lengths are small, however, good LDPC codes become more difficult to find using these random construction methods [11].

A key idea in this paper is that the absence of cycles of length 4 in the Tanner graph associated with an LDPC code can be systematically avoided by taking as parity-check matrices the incidence matrices of suitably chosen combinatorial designs. For small block lengths in particular, an analytic construction method that guarantees

1. small, uniform row and column weights; and

2. the absence of 4-cycles,

is expected to be particularly useful.

Selecting (3,6)-regular LDPC codes in particular can provide an advantage in terms of encoding complexity. Richardson and Urbanke [5] have recently shown that the actual number of operations required to encode $(3,6)$-regular codes is no more than $0.017^{2} n^{2}$.

In this paper we present a construction for a family of paritycheck matrices having column weight 3 , and that satisfy both items 1 and 2 in order to provide a useful analytic construction method for regular LDPC codes. As our construction is based on combinatorial design theory, we present in Section II of this paper some background material on designs before describing the constructions and some of their properties in Section III. Section IV details the performance of our LDPC codes when belief propagation decoding is used. Section V concludes the body of the paper, and explicit constructions of Kirkman triple systems are presented in the Appendix. 


\section{COMbinatorial DEsignS}

A combinatorial design is an arrangement of a set of $v$ points into $b$ subsets, called blocks, which satisfy certain regularity conditions. A design is regular if the number of points in each block, and the number of blocks which contain each point are the same for every point and block in the design, designated $\gamma$ and $\rho$ respectively. The covalency $\lambda_{x y}$ of the points $x$ and $y$ is the number of blocks that contain them both. A design is balanced if $\lambda_{x y}$ is a constant for all $x$ and $y$, the covalency of the design is then $\lambda$. A regular balanced design is often denoted as a $(v, b, \rho, \gamma, \lambda)$-design.

Every design can be described by a $b \times v$ incidence matrix $I$ where each row in $I$ represents a block $B_{i}$ of the design and each column a point $P_{j}$ :

$$
I_{i, j}= \begin{cases}1 & \text { if } P_{j} \in B_{i} \\ 0 & \text { otherwise }\end{cases}
$$

For a regular design the number of ones in $I$ is

$$
v \times \rho=b \times \gamma
$$

The incidence matrix of a combinatorial design, or its transpose, can be used as the parity-check matrix of a binary LDPC code to give favorable properties to the code. The transpose of the incidence matrix of a $(v, b, \rho, \gamma, \lambda)$-design will give an $v \times b$ parity-check matrix $H$ having $v$ parity-check equations, block length $n=b$, row weight $\rho$ and column weight $\gamma$. Choosing a design with $\lambda=0$ or 1 guarantees the absence of 4-cycles in the code. As is the case for random constructions of paritycheck matrices, the $H$ constructed in this way are not necessarily full rank in which case the number of message bits in the code is $n-\operatorname{rank}(H)$.

One class of combinatorial designs that have already been examined for generating LDPC codes are Steiner triple systems (STS), or $(v, b, \rho, 3,1)$-designs [11]. These designs exist for all $v \equiv 1,3(\bmod 6)$, are regular with column weight 3 , row weight $(v-1) / 2$, and are free of 4 -cycles. STS-LDPC codes have as their parity check matrix the transpose of the STS incidence matrix. These codes have codeword length $n=m(m-1) / 6$, where $m=v$ and are high rate codes. If the restriction that $\lambda=1$ is relaxed to allow $\lambda=1$ or 0 blocklength and hence rate can be reduced. A simplistic approach is to remove some columns of $H$; for each pair of points in the omitted column the corresponding $\lambda_{x y}$ will be 0 . However, this results in a parity-check matrix with variable row weights, which can be as low as 1 or 0 , leading to performance penalties when iteratively decoded.

The key idea presented in this paper is to use a class of designs called Kirkman triple systems (KTS) to derive regular $(v, b, \rho, 3,\{1,0\})$-designs. Kirkman triple systems are defined as the resolvable Steiner triple systems. That is, the blocks of a Kirkman triple system can be arranged into $\rho$ groups such that the $\frac{v}{3}$ blocks of each group are disjoint, and each group contains every point precisely once. Consequently, if all blocks in a group are removed from $H$ what remains is a parity-check matrix $H_{0}$ with $v$ parity checks, row weight $\rho-1$ and $n=b-\frac{v}{3}$. If we retain the blocks from just six groups, for example, the result is a $(3,6)$-regular code without 4 -cycles. (As there were no 4 -cycles in the original $H$ removing columns cannot add any).

In general, we can take any KTS and use one or more of its groups to make up our parity-check matrix. The resulting $H$ has the same number of parity-checks as the original, still has column weight 3 and no 4 -cycles, but can have any desired row weight $\rho \in\{1,2, \ldots,(v-1) / 2\}$. The number of code bits is then $n=\frac{\rho v}{3}$ as per equation (1). Kirkman triple systems exist for all $v \equiv 3(\bmod 6)$ [12]. Construction methods for $v=3 q$ and $v=2 q+1, q$ a prime power, are given in [13], and presented in the Appendix.

\section{LDPC CODES FROM COMBINATORIAL DESIGNS}

In this section we apply combinatorial designs to construct LDPC codes. Two designs are isomorphic if there exists a oneto-one mapping between their incidence matrices, that is, the incidence matrix of one can be obtained via column and row permutations of the other. There are 80 non-isomorphic STS $(35,15,7,3,1)$-designs, and while it is not known exactly how many non-isomorphic designs exist for larger Steiner triple systems, the number rapidly increases with $v$. The vast majority of the non-isomorphic Steiner triple systems for any given design are full rank [14]. Thus when considering a particular code we have assumed a full rank design has been chosen and therefore the rate of the code is $\frac{n-m}{n}$.

In addition to Steiner and Kirkman triple systems we examine:

- Codes from binary Euclidean geometries (EG-LDPC), $\left(q^{2}-\right.$ $\left.1, q^{2}-1, q, q, 1\right)$-designs, and codes from binary projective geometries (PG-LDPC), $\left(q^{2}+q+1, q^{2}+q+1, q+1, q+1,1\right)$ designs, $q=2^{s}, s$ any integer, have been investigated in [7]. PG and EG designs produce square incidence matrices, however previous results have established a combinatorial expression for the rank of $H$ and hence the rate of these codes [15].

- A construction for LDPC codes using Ramanujan graphs is presented in [16]. The advantage of this method of construction is that it produces $(3,6)$-regular codes. However these codes can only be constructed for block lengths $n=2\left(q^{3}-q\right)$ where $q$ is prime.

From Fig. 1, one advantage of using Kirkman triple systems to construct $(3, \rho)$-regular LDPC codes is the wealth of code rates and block lengths that are available. Although methods such as puncturing or extending existing parity check matrices [23] can be used to create additional LDPC codes, only analytically constructed codes are presented in Fig. 1.

\section{A. Girth}

A simple lower bound on the girth of an LDPC code can be found by considering the associated Tanner graph. Our line of reasoning is similar to, though extends, [16, ,Lemma 1] relating 


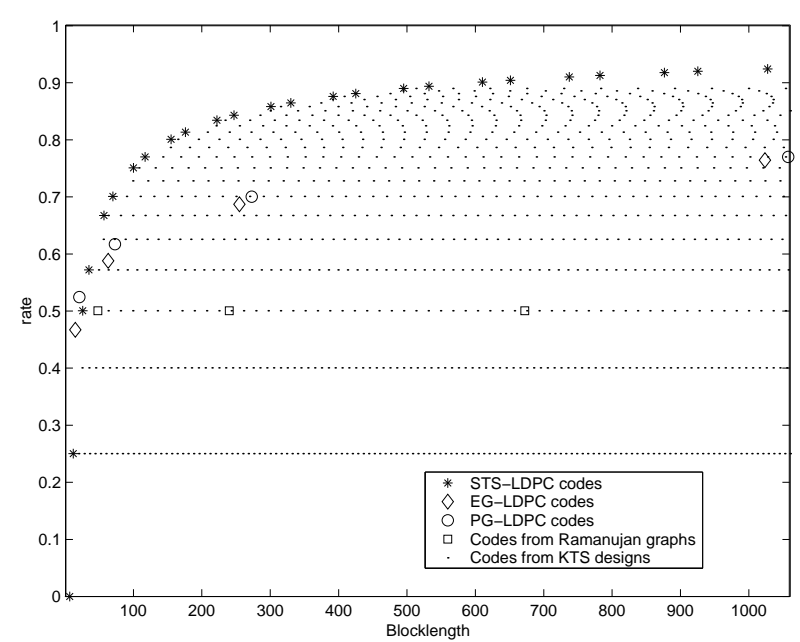

Fig. 1. Blocklength and rate of analytically constructed LDPC codes

the girth of codes from Ramanujan graphs to block length for any girth $\equiv 2(\bmod 4)$.

Consider a parity check matrix $H=I^{T}$ where $I$ is the incidence matrix of a regular combinatorial design. Take an arbitrary bit vertex $n_{1}$ which is connected to $\gamma$ parity-check vertices. Each of these is in turn connected to $\rho-1$ bit vertices other than $n_{1}$. If any of these $\gamma(\rho-1)$ bit vertices are the same a 4 -cycle results. (Say checks $p_{1}$ and $p_{2}$ on $n_{1}$ both contain bit $n_{2}$ then the edges from $n_{1}$ to $p_{1}$ to $p_{2}$ to $n_{2}$ and back to $n_{1}$ form a cycle containing 4 edges). Thus to avoid 4 -cycles there must be at least $n \geq \gamma(\rho-1)+1$ code word bits, so no two parity-checks on $n_{1}$ must share any.

Now consider the $\gamma(\rho-1)$ bit vertices above; each is connected to a further $\gamma-1$ parity-check vertices. To avoid both 4 - and 6-cycles these $\gamma(\rho-1)(\gamma-1)$ vertices and the $\gamma$ other parity-check vertices already connected to $n_{1}$ must be distinct. Thus to avoid 6-cycles $m \geq \gamma(\rho-1)(\gamma-1)+\gamma$. Similarly we can start with an arbitrary parity-check vertex $p_{1}$, and follow the reasoning above to get the following restrictions: To avoid 4-cycles, $m \geq \rho(\gamma-1)+1$. To avoid 6-cycles, $n \geq \rho(\gamma-1)(\rho-1)+\rho$.

This reasoning can be extended to any cycle size $c$ to obtain the following relationship between the block length $n$ needed to avoid a cycle size $c$, and the parity-check and codeword bit degrees, $\gamma$ and $\rho$, respectively:

$$
\begin{gathered}
n \geq 1+\gamma(\rho-1)+\cdots+\gamma(\rho-1) \alpha^{\frac{c}{4}-1}, \\
c=0(\bmod 4), \\
n \geq \rho+\rho \alpha+\cdots+\rho \alpha^{\frac{-2 c}{4}}, \\
c=2(\bmod 4),
\end{gathered}
$$

where $\alpha=(\gamma-1)(\rho-1)$.

The inequalities in (2), and in particular the restriction on $n$ to avoid 6-cycles, can be used to prove the following Lemma.

Lemma 1: The girth of any STS-LDPC, EG-LDPC, or PGLDPC code is 6 .
Proof: In each case, we use the appropriate design parameters and substitute into equation (2) for $c=6$.

Using the properties of STS designs, $\gamma=3, \rho=(m-1) / 2$, and $n=m(m-1) / 6$, we obtain an inequality in $m$ :

$$
\frac{m(m-1)}{6} \geq 2 \frac{(m-1)}{2}\left[\frac{m-1}{2}-1\right]+\frac{m-1}{2}
$$

or, equivalently, $m \leq 3$. So for any $m>3,6$-cycles must exist in STS-LDPC codes. Further, for $m=3$, the blocklength is 1 , which is not a valid code.

Using the properties of projective geometries, $\rho=\gamma=q+1$, and $n=q^{2}+q+1$ we obtain an inequality in $q$ :

$$
q^{2}+q+1 \geq(q+1) q^{2}+(q+1)
$$

so that $q \leq 0$, which cannot be met for any prime $q$, so 6 -cycles must exist in all PG-LDPC codes.

Finally, using the properties of Euclidean geometries, $\rho=$ $\gamma=q$, and $n=q^{2}-1$ we obtain an inequality in $q$ :

$$
q^{2}-1 \geq q(q-1)(q-1)+q,
$$

so that

$$
q\left(q^{2}+2\right)+1 \leq 3 q^{2},
$$

which cannot be met for any prime $q$, hence 6 -cycles must exist in all EG-LDPC codes. Further, the existence of cycles smaller than 6 in each of the above codes are excluded by the restriction that $\lambda=0$ or 1 and the result follows.

No such bounds can be placed on the regular codes derived from Kirkman triple systems, or the regular codes derived from Ramanujan graphs. These codes have constant row and column weight as $n$ increases and so their density decreases allowing the girth to grow with $n$.

\section{B. Minimum distance}

For regular LDPC codes whose parity-check matrix is the incidence matrix of a Steiner triple system, MacKay and Davey [17, Theorem 1] showed that the minimum distance is at most 10. While this upper bound on minimum distance is so small as to preclude the use of Steiner triple system LDPCs for all but the shortest block lengths, it is possible to systematically construct STS LDPCs having minimum distance at least 6 for all $v$ for which $\operatorname{STS}(v)$ exist except for $v=7$ or 13 , where the minimum distance is 4 .

To see this, recall that the minimum distance of a code is equal to the minimum nonzero number of columns in the parity-check matrix for which a nontrivial linear combination sums to zero [18, p. 84]. The definition of Steiner triple systems ensures that all columns in the parity-check matrix have weight 3 , and that no two columns share more than one point. Therefore at least 4 columns are needed to sum to zero.

To show that the minimum distance is at least 6 , we need to establish the existence of Steiner triple systems that lack collections of 4 blocks employing just 6 points. Collections of 
blocks in incidence structures are referred to as configurations. The particular configuration consisting of just 4 blocks and 6 points, with each block containing 3 points, and each point incident with precisely 2 blocks is called a Pasch configuration, or quadrilateral. The term anti-Pasch is used to describe a Steiner triple systems that lacks a Pasch configuration.

It is well known that Steiner triple systems of order $m$ exist if and only if $v \equiv 1,3(\bmod 6)$, and it had been a long standing conjecture that anti-Pasch Steiner triple systems also exist for all these values except for $v=7$ or 13 , for which they are known not to exist. After some twenty years the conjecture has recently been solved [19], [20]. We therefore have the following result, and refer the reader to the papers [19], [20] for explicit constructions of the associated anti-Pasch Steiner triple systems.

Lemma 2: For all $v \equiv 1,3(\bmod 6)$ except for $v=7,13$, there exist regular LDPC codes of length $v(v-1) / 6$, having parity check matrices with uniform column weight 3 , uniform row weight $(v-1) / 2$ and minimum distance at least 6 .

The minimum distance of the $(3, \rho)$-regular codes described above can be no smaller than that of the resolvable STS codes from which they are constructed (removing columns cannot reduce, but can increase, the size of the linear combination of columns that sum to zero). However, as yet we have no explicit determination of the minimum distance of the $(3, \rho)$ regular codes presented here. It is worth noting that resolvable $(v, b, \rho, \gamma, 1)$ designs exist for $\gamma \in\{4,5, \ldots\}$ and that these designs would result in codes with minimum distance $d=\{5,6, \ldots\}$. However, simulation results suggest a performance degradation when the column weights of resolvable designs and randomly constructed codes are increased from 3 to 4 .

\section{Simulation RESUlts USING ITERATIVE DECODING}

We employed belief propagation decoding, also known as sum-product decoding, as presented in [7]. In the simulation results that follow, a number of randomly generated LDPC codes have been used. Where possible we have used codes already published [6], [21]. Where none were available we have used the best random construction we could generate using the following construction method [2], [22]:

- $\gamma$ ones are placed in each column of $H$ with an attempt made to keep the number of ones in each row approximately the same.

- extra ones are randomly added so that the weight of each row is greater than one.

- if the column weights of $H$ are all even, further ones are added to positions selected randomly from the entire matrix (it is undesirable for the sum of the rows to be weight one or less) - to remove 4-cycles, ones are moved randomly within columns involved in the cycle.

Fig. 2 shows the performance of rate half KTS and randomly generated LDPC codes. For the codeword length $n \approx 500$ we have found a randomly constructed (nearly regular) LDPC

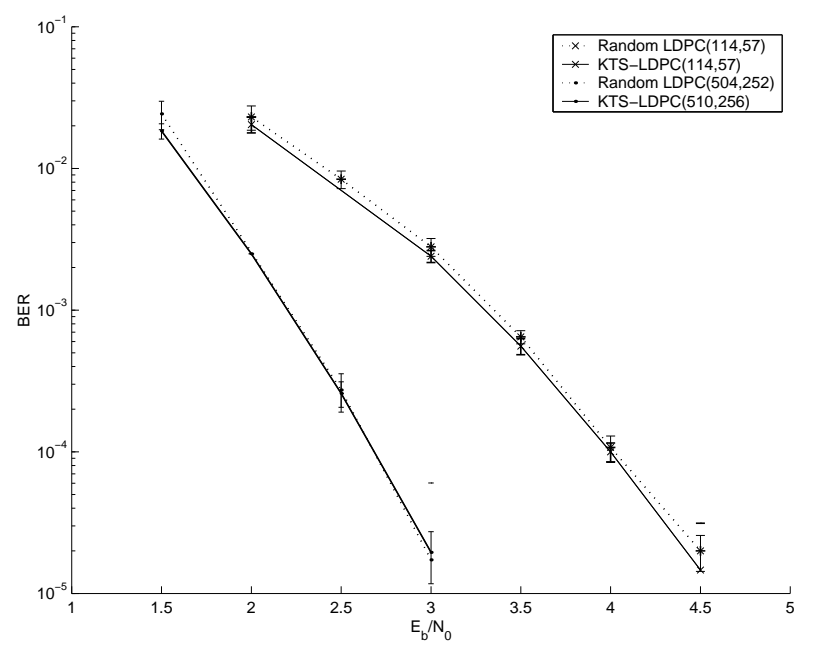

Fig. 2. BER vs. $E_{b} / N_{0}$ for rate $1 / 2$ LDPC codes, $\max$. iterations $=500$, 0.99 confidence intervals shown

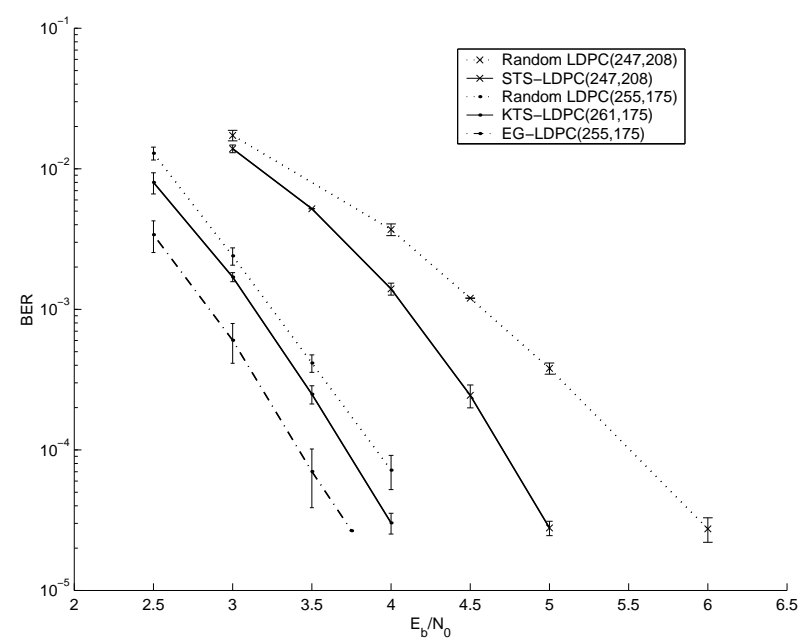

Fig. 3. BER vs. $E_{b} / N_{0}$ for high-rate LDPC codes, max. iterations $=50,0.99$ confidence intervals shown

code which performs as well as the regular code constructed from Kirkman triple systems. However for a smaller codeword length $n \approx 100$ the KTS derived system performs better.

The leftmost curves in Fig. 3 show the performance of rate $2 / 3$ KTS, EG and randomly generated LDPC codes. The LDPC code generated from Kirkman triple systems is a $(3,9)$ regular code, the EG code is $(16,16)$-regular, and the randomly generated LDPC code has row weights between 7 and 12, and constant column weight 3 . While all three codes have similar block lengths and rates the EG code has more than five times as many non zero entries in its parity-check matrix, resulting in a significant increase in computational complexity for the same number of decoding iterations.

The two length $n=247$ codes in Fig. 3 have the same rate and density of $H$. Using the random construction method we were unable to eliminate 4 -cycles from the high rate $n=247$ code. This is an advantage of our analytically constructed 
codes over random constructions of high rate codes.

MacKay and Davey rejected Steiner triple systems as LDPC codes due to their poor minimum distance properties [11]. While they do have poor minimum distances our results suggest that their good girth properties compensate for this when belief propagation decoding is used and $n$ is small. The $(3, \rho)$ regular codes derived from Kirkman triple systems do not have the minimum distance constraints of the STS codes and have the additional advantage that they can improve upon their good girth properties. Even for large $n$ the $(3, \rho)$-regular codes perform as well as randomly constructed codes with the added advantage that they are regular which may reduce the complexity of encoding, decoding and storage in an implementation of LDPC codes.

\section{CONCLUSiON}

We have presented a construction method for LDPC codes that produces parity-check matrices having constant column and row weight and girth of at least 6 . These $(3, \rho)$-regular codes can be constructed for any number of parity-check sums $m \equiv 3(\bmod 6)$, and for all row weights $\rho \in\{1,2, \ldots,(m-$ $1) / 2\}$. Their regularity and ease of construction may prove an advantage over randomly constructed codes. The construction is particularly useful for small block lengths, and those high-rate codes for which random construction methods have difficulty removing 4-cycles.

\section{APPENDIX}

\section{CONSTRUCTING KIRKMAN TRIPLE SYSTEMS [13]}

Let $q=6 m+1$ be a prime power, $m$ any integer, then a Kirkman triple system with $v=3 q$ exists.

Construction: take $\theta$, a primitive element of $\operatorname{GF}(q)$, so that $\theta^{6 m}=1, \theta^{3 m}=-1$, and $\theta^{2 m}+1=\theta^{m}$. Then use three copies of each element of $\operatorname{GF}(q)$ to construct the sets

$$
\begin{aligned}
& A=\left\{0_{1}, 0_{2}, 0_{3}\right\} \\
& B_{i j}=\left\{\theta_{j}^{i}, \theta_{j}^{i+2 m}, \theta_{j}^{i+4 m}\right\}, 1 \leq i \leq m, 1 \leq j \leq 3 \\
& C_{i j}=\left\{\theta_{j}^{i+m}, \theta_{j+1}^{i+3 m}, \theta_{j+2}^{i+5 m}\right\}, 1 \leq i \leq m, 1 \leq j \leq 3(\bmod 3) \\
& D_{i j}=\left\{\theta_{j}^{i}, \theta_{j+1}^{i+2 m}, \theta_{j+2}^{i+4 m}\right\}, 1 \leq i \leq m, 1 \leq j \leq 3(\bmod 3)
\end{aligned}
$$

The sets $A, B_{i j}, C_{i j},(1 \leq i \leq m, 1 \leq j \leq 3)$ form one resolution class, and the translates of this class give a further $6 \mathrm{~m}$ classes. Next, each $D_{i j}$ with its translates give a further resolution class; so we obtain a total of $9 m+1$ resolution classes.

Let $q=6 m+1$ be a prime power, $m$ any integer, then a Kirkman triple system with $v=2 q+1$ exists.

Construction: Take $\theta$, a primitive element of $\operatorname{GF}(q)$, so that $\theta^{6 m}=1, \theta^{3 m}=-1$, and choose $u$ so that $\theta^{m}+1=2 \theta^{u}$. Then use two copies of each element of $\operatorname{GF}(q)$ and, with an extra element $\infty$, construct the sets

$$
\begin{aligned}
& A=\left\{0_{1}, 0_{2}, \infty\right\} \\
& B_{i}=\left\{\theta_{2}^{i+u+m}, \theta_{2}^{i+u+3 m}, \theta_{2}^{i+u+5 m}\right\}, \quad 0 \leq i \leq m-1 \\
& C_{i}=\left\{\theta_{1}^{i}, \theta_{1}^{i+m}, \theta_{2}^{i+u}\right\}, \quad 0 \leq i \leq m-1 \\
& D_{i}=\left\{\theta_{2}^{i+2 m+u}, \theta_{1}^{i+2 m}, \theta_{1}^{i+3 m}\right\}, \quad 0 \leq i \leq m-1 \\
& E_{i}=\left\{\theta_{2}^{i+4 m+u}, \theta_{1}^{i+4 m}, \theta_{1}^{i+5 m}\right\}, \quad 0 \leq i \leq m-1 .
\end{aligned}
$$

The sets $A, B_{i}, C_{i}, D_{i}, E_{i},(0 \leq i \leq m-1)$ make up the first resolution class, and the translates of this class give a further $q-1$ classes.

\section{REFERENCES}

[1] R. G. Gallager, "Low-density parity-check codes," IRE Trans. Information Theory, vol. IT-8, no. 1, pp. 21-28, January 1962.

[2] D. J. C. MacKay and R. M. Neal, "Near Shannon limit performance of low density parity check codes," Electronics Letters, vol. 32, no. 18, pp. 1645-1646, March 1997.

[3] R. M. Tanner, "A recursive approach to low complexity codes," IEEE Trans. Inform. Theory, vol. IT-27, no. 5, pp. 533-547, September 1981.

[4] R. G. Gallager, Low Density Parity Check Codes, no. 21 in Research Monograph Series. Cambridge, MA: MIT Press, 1963.

[5] T. Richardson and R. Urbanke, "Efficient encoding of low-density paritycheck codes," IEEE Transactions on Information Theory, vol. 47, no. 2, pp. 638-656, February 2001.

[6] D. J. C. MacKay, "Good error-correcting codes based on very sparse matrices," IEEE Trans. Inform. Theory, vol. 45, no. 2, pp. 399-431, March 1999

[7] R. Lucas, M. P. C. Fossorier, Y. Kou, and S. Lin, "Iterative decoding of one-step majority logic decodable codes based on belief propagation," IEEE Trans. Commun., vol. 48, no. 6, pp. 931-937, June 2000.

[8] R. J. McEliece, D. J. C. MacKay, and J.-F. Cheng, "Turbo decoding as an instance of Pearl's "belief propagation" algorithm," IEEE J. Selected Areas Commun., vol. 16, no. 2, pp. 140-152, February 1998.

[9] T. Etzion, A. Trachtenberg, and A. Vardy, "Which codes have cyclefree Tanner graphs?," IEEE Trans. Inform. Theory, vol. 45, no. 6, pp. 2173-2181, September 1999.

[10] V. Sorokine, F. R. Kschischang, and S. Pasupathy, "Gallager codes for CDMA applications-Part I: Generalizations, constructions, and performance bounds," IEEE Trans. Commun., vol. 48, no. 10, pp. 1660-1668, October 2000.

[11] D. J. MacKay and M. C. Davey, "Evaluation of Gallager codes for short block length and high rate applications," Proceedings of the IMA Workshop on Codes, Systems and Graphical Models, 1999.

[12] D. K. Ray-Chaudhuri and R. M. Wilson, "Solution of Kirkmans schoolgirl problem,” Proc. Symp. Math., vol. 19, pp. 187-203, 1971.

[13] I. Anderson, Combinatorial Designs: Construction Methods, Mathematics and its Applications. Ellis Horwood, Chichester, 1990.

[14] E. F. Assmus, Jr., "On 2-ranks of Steiner triple systems," Electron. J. Combinatorics, vol. 2, pp. Research paper \#R9, 1995.

[15] S. Lin, "On the number of information symbols in polynomial codes," IEEE Trans. Inform. Theory, vol. IEEE Trans. Inform. Theory, no. IT-18, pp. 785-794, November 1972.

[16] J. Rosenthal and P. O. Vontobel, "Constructions of LDPC codes using Ramanujan graphs and ideas from Margulis," Proc. 38th Allerton Conference on Communications, Control and Computing., October 2000.

[17] D. J. C. MacKay and M. C. Davey, "Evaluation of Gallager codes for short block length and high rate applications," in Codes, Systems and Graphical Models; volume 123 of IMA Volumes in Mathematics and its Applications;, B. Marcus and J. Rosenthal, Eds., pp. 113-130. SpringerVerlag, New York, 2000.

[18] S. B. Wicker, Error Control Systems for Digital Communication and Storage, Prentice-Hall, Upper Saddle River, NJ 07458, 1995.

[19] A. C. H. Ling, C. J. Colbourn, M. J. Grannell, and T. S. Griggs, "Construction techniques for anti-Pasch Steiner triple systems," J. Lond. Math. Soc., vol. 61, no. 3, pp. 641-657, June 2000.

[20] M. J. Grannell, T. S. Griggs, and C. A. Whitehead, "The resolution of the anti-Pasch conjecture," J. Combin. Designs, vol. 8, no. 4, pp. 300-309, July 2000.

[21] D. J. MacKay, ," http://wol.ra.phy.cam.ac.uk/mackay/.

[22] R. M. Neal, ," http://www.cs.toronto.edu/ radford/homepage.html.

[23] Y. Kou, S. Lin, and M. Fossorier, "Low density parity check codes: Construction based on finite geometries", in Proc. IEEE Globecom Conf., San Francisco, CA, November 2000, pp. 825-829. 